\title{
Precise Isotopic Measurements of Nitrogen at the Sub-Nanomole Level
}

\author{
Naoto TaKahata*, Yoshiro Nishio**, Naohiro YoshidA*** and Yuji SANO* \\ *Department of Earth and Planetary Sciences, Faculty of Science, Hiroshima University, \\ Kagamiyama, Higashi Hiroshima 739-8526, Japan \\ **Geological Institute, School of Science, The University of Tokyo, \\ Bunkyo, Tokyo 113-0033, Japan \\ ***Institute for Hydrospheric-Atmospheric Sciences, Nagoya University, \\ Chikusa, Nagoya 464-8602, Japan
}

\begin{abstract}
A system for the isotopic measurement of nitrogen at the sub-nanomole level has been developed using a modified noble gas mass spectrometer, allowing ${ }^{40} \mathrm{Ar} /{ }^{36} \mathrm{Ar}, \mathrm{N}_{2} /{ }^{40} \mathrm{Ar},{ }^{4} \mathrm{He} /{ }^{40} \mathrm{Ar}$ and $\mathrm{C} / \mathrm{N}$ ratios as well as the $\delta^{15} \mathrm{~N}$ value to be measured on one sample. Moreover, carbon dioxide can be collected for a $\delta^{13} \mathrm{C}$ measurement. The experimental details concerning the analytical system are presented here, including the nitrogen purification procedure and its performance. The analytical errors concerning the $\delta^{15} \mathrm{~N}$ values and ${ }^{28} \mathrm{~N}_{2} /{ }^{40} \mathrm{Ar}$ ratios were estimated to be about $0.3 \% \circ$ and $3 \%$ for actual samples. Using six basalt glasses, the ability of the instrument to make accurate nitrogen isotope measurements has been evaluated. The observed values of the nitrogen content, nitrogen isotopes and nitrogen/argon ratios are compatible with the data in the literature. The results of some basic experiments and the observation of basalt glasses demonstrate that the new system can be applied to measuring nitrogen isotopes in geochemical and environmental samples.
\end{abstract}

Keywords $\delta^{15} \mathrm{~N}$, nitrogen isotope, argon isotope, $\mathrm{N}_{2} /{ }^{40} \mathrm{Ar}$ ratio, sub-nanomole

Although nitrogen constitutes the major portion of the Earth's atmosphere, its degassing from the mantle and its circulation in the mantle are not well known. The reason is that because of a $<1 \mathrm{nmol} / \mathrm{g}-\mathrm{N}$ concentration, the nitrogen isotope ratios contained in mantle-derived materials, such as mid-ocean-ridge basalt glasses and diamonds, have been difficult to measure precisely using conventional dynamic mass spectrometers. Recently, Wright et al. ${ }^{1}$ established a technique that enabled nitrogen isotopic ratios on sub-nanomole level samples to be precisely measured by using a high-vacuum line used for gas extraction and purification, and a modified noble gas mass spectrometer. Subsequently, studies of isotopic sub-nanomole quantities of nitrogen in terrestrial samples by a similar measurement technique have been reported. ${ }^{2,3}$ In order to discuss the origin of nitrogen, noble gas data (especially argon isotopes) are particularly useful. ${ }^{4}$ Therefore, we have developed a system that enables the measurement of multiple elements simultaneously, without compromising the precision of the nitrogen isotopes.

In this paper we give an outline of the system, and its performance by using the results of some basic experiments, and then measurements of nitrogen isotopes in reference mid-ocean ridge basalt glasses (which are already reported ${ }^{2}$ ) in order to evaluate the usefulness of the new system.

\section{Experimental}

\section{Purification and separation of nitrogen and argon}

Since nitrogen constitutes the major portion of the atmosphere, one must be careful of atmospheric contamination during isotopic and abundance measurements. Also, carbon monoxide and hydrocarbons contained in samples such as volcanic gases and rocks must be fully removed, because they are the major interfering species for nitrogen measurements at masses of 29 and 28. To solve this problem, we have developed a low-background vacuum line for the separation and purification of nitrogen at a sub-nanomole level.

Figure 1 shows a schematic diagram of the separation and purification of nitrogen and noble gases. Figure 2 shows an outline of the high-vacuum line used for nitrogen and noble gas extraction, purification and separation from rock and gas samples. The extraction, purification and separation lines are basically made of stainless steel. Pyrex glass tubes are used for cold traps (CT), since stainless steel adsorbs a considerable amount of nitrogen on its surface at liquid-nitrogen boiling temperature. Quartz glass tubes are used for the $\mathrm{CuO}$ finger and $\mathrm{Pt}$ finger because high temperatures are experienced.

The vacuum line is basically separated into five parts by all-metal bellow valves (Va, V1, V2 and Vc), two 


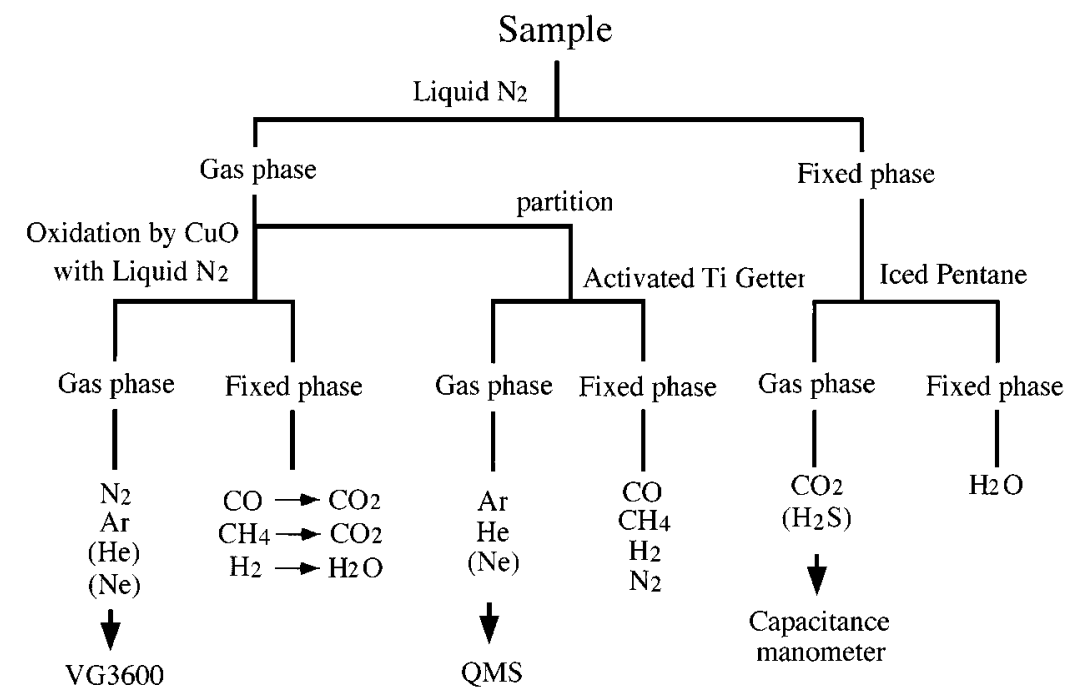

Fig. 1 Diagram of the nitrogen and argon purification procedure.

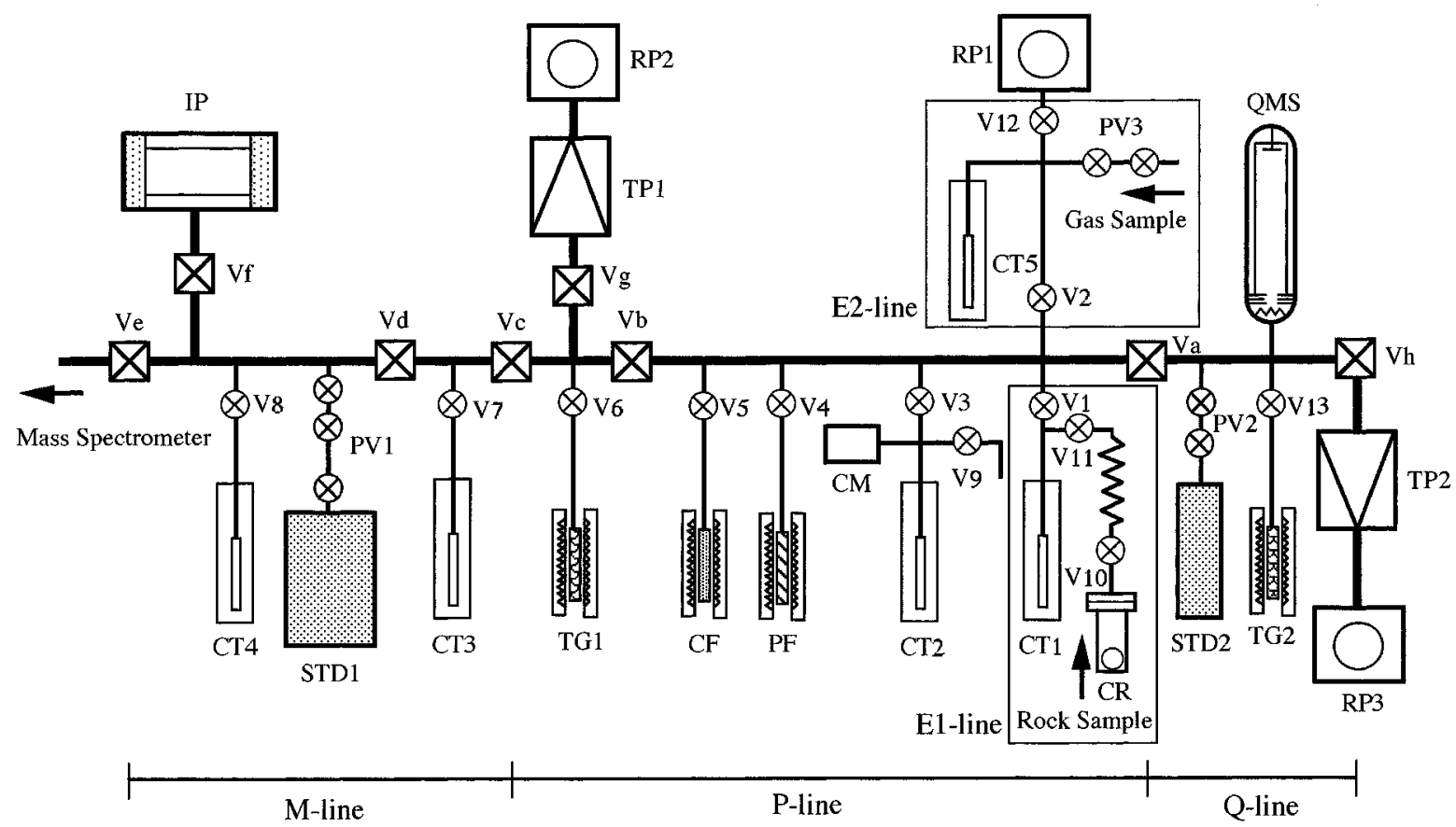

Fig. 2 Nitrogen and argon purification vacuum line. QMS, quadrupole mass spectrometer; TP, turbo molecular pump; RP, oil rotary pump; CM, capacitans manometer; CT, cold trap; PF, platinum foil; CF, copper oxide finger; TG, titanium getter; STD, standard gas reservoir; CR, crusher; PV, pipette valve; $\mathrm{V} \& \mathrm{v}$, valve.

gas-extraction lines (E1-line, E2-line), a gas-purification line (P-line), a vacuum line directly connected to the mass spectrometer (M-line) and a vacuum line for measurements by a quadrupole mass spectrometer (Qline). The E-line and P-line are evacuated by a turbo molecular pump (TP1) down to $10^{-7}$ Torr. The M-line and Q-line are evacuated by an ion sputter pump (IP) and another turbo molecular pump (TP2) down to $10^{-8}$ and $10^{-7}$ Torr, respectively. This vacuum line is similar to that already reported. ${ }^{5}$ However, our system uses a sector type mass spectrometer for nitrogen measure- ments and a ball mill type crusher (CR) for gas extraction from rock samples. We adopted the vacuum crushing technique for nitrogen extraction, instead of the conventional stepped heating one. This is because nitrogen extracted by crushing is more certain to be indigenous to the sample than that obtained by heating. ${ }^{4}$ In other words, by the crushing technique the contribution of contaminations of adsorbed air and organic materials sticking on grain surfaces can be minimized.

A rock sample is introduced into a crusher $(\mathrm{CR})$ and the E1-line is evacuated. The weight of the chips of 
rock used is about $2 \mathrm{~g}$, and usually provides a sufficient quantity of nitrogen for isotopic measurements, since the mass spectrometer is capable of measuring nitrogen isotopes in as little as $0.1 \mathrm{nmol}$ of gas. Before an analysis, a helium leak check is made using a quadrupole mass spectrometer. Then, the crusher is baked out at $150^{\circ} \mathrm{C}$ overnight to desorb any atmospheric nitrogen. At the same time, the E1-line and the P-line are baked at $250^{\circ} \mathrm{C}$ to degass the contaminant gases.

Before the experiment, the crusher and the vacuum lines are cooled down to room temperature. Then, V10 is closed to isolate the sample from the vacuum and the sample is crushed using a ball mill (CR). After crushing for $5 \mathrm{~min}$, the extracted gas is transferred to a first purification section (E1-line) by opening V10.

At first, carbon dioxide, water vapor and the other condensable gases are separated by a cold trap (CT1) kept at liquid-nitrogen boiling temperature. Then, the non-condensable gases are admitted into the purification line (P-line) by opening V1. The expanded gases are divided into a nitrogen fraction and a noble gas fraction. The gases in the E-line and the P-line are allocated for a nitrogen measurement and a noble gas measurement, respectively. After $5 \mathrm{~min}, \mathrm{~V} 1$ is closed so as to divide the two fractions. At this time the pressure in the P-line is checked by a capacitance manometer (CM). Pirani, shultz and ionization guages are not used because they might affect the nitrogen isotope ratio by ionizing the sample gas.

Then, the gases in the P-line are reacted for $5 \mathrm{~min}$ with pure $\mathrm{O}_{2}$ produced by heating the copper oxide finger $(\mathrm{CF})$ at $850^{\circ} \mathrm{C}$. This is carried out in the presence of platinum foil $(\mathrm{PF})$ at $1000^{\circ} \mathrm{C}$. At this time carbonaceous gases, such as $\mathrm{CO}, \mathrm{CH}_{4}$ and higher hydrocarbons, are oxidized to $\mathrm{CO}_{2}$, and hydrogen is converted to $\mathrm{H}_{2} \mathrm{O}$ and sulfur to $\mathrm{SO}_{2}$. After $5 \mathrm{~min}$, excess oxygen is resorbed onto the copper oxide, first at $600^{\circ} \mathrm{C}$ for $5 \mathrm{~min}$ and then at $450^{\circ} \mathrm{C}$ for $10 \mathrm{~min}$, during which time the condensible gases, such as $\mathrm{CO}_{2}, \mathrm{SO}_{2}$ and $\mathrm{H}_{2} \mathrm{O}$, are adsorbed in a cold trap (CT2) held at liquid-nitrogen boiling temperature. Purified gases are introduced into the M-line by opening $\mathrm{Vc}$, and impurities are further adsorbed in the cold traps (CT3, CT4) held at liquidnitrogen boiling temperature.

The quantity of purified nitrogen is measured by a quadrupole mass spectrometer (QMS; Massmate-100, ULVAC). The part of purified gas in the P-line is introduced into the Q-line. The remains are used to measure nitrogen isotopes, because the gases in the Qline may be affected on the nitrogen isotope ratio due to isotopic fractionation. In order to balance the size of the sample nitrogen with that of standard nitrogen (STD1), the sample size introduced into a mass spectrometer can be reduced by repeated expansion processes. Finally the purified gas in the part of the Mline (between $\mathrm{Vd}$ and $\mathrm{Ve}$ ) is admitted into a mass spectrometer.

The nitrogen standard is reserved in a large metal container (STD1). The standard gas is introduced into the M-line by using a pipette valve (PV1), which can adjust the amount of standard gas. The standard gas contains almost pure nitrogen and a little argon. The standard gas is purified by using only a cold trap (CT4) held at liquid-nitrogen boiling temperature. The nitrogen isotope ratio and ${ }^{28} \mathrm{~N}_{2} /{ }^{40} \mathrm{Ar}$ ratio of the standard gas are measured before and after the sample analysis. The isotopic composition of the standard gas is periodically checked by a comparison with that of the local air.

After the purified nitrogen of the sample in the Mline has been sent to the mass spectrometer, the remaining gas in the P-line and Q-line are all evacuated by two turbo molecular pumps (TP1, TP2), respectively. Then, the noble gas fraction in the E1-line is introduced into the P-line by opening V1. After $5 \mathrm{~min} \mathrm{V1}$ is closed, and chemically active gases, including nitrogen, in the P-line are all absorbed by a hot titanium getter (TG1). The temperature of the getter is cooled from $700^{\circ} \mathrm{C}$ to $500^{\circ} \mathrm{C}$ over $10 \mathrm{~min}$. After the size of the sample noble gas is checked by a capacitance manometer, the purified gas is admitted into the Q-line, and argon isotope ratio and ${ }^{4} \mathrm{He} /{ }^{40} \mathrm{Ar}$ ratio are measured by a quadrupole mass spectrometer using another titanium getter (TG2) at room temperature. The measurement is carried out in a static mode.

The in-house mixed gas (Nippon Sanso Co.) is kept as the noble gas standard in another large-volume metal container (STD2). Standard gas is introduced into the Q-line by using a pipette valve (PV2). The standard gas contains $\mathrm{He}, \mathrm{Ne}$ and $\mathrm{Kr}$ at $1 \%$ and $\mathrm{Ar}$ at $97 \%$ (volume). The argon isotope ratio and ${ }^{4} \mathrm{He} /{ }^{40} \mathrm{Ar}$ ratio of the standard gas are measured before and after the sample analysis. The isotopic composition of the standard gas is periodically checked by a comparison with the gas collected in a bottle whose argon isotope ratio and ${ }^{4} \mathrm{He} /{ }^{40} \mathrm{Ar}$ ratio are known.

After the noble gas measurement has been made, the remaining gas in the $\mathrm{P}$-line and Q-line are all evacuated by two turbo molecular pumps (TP1, TP2), respectively. At the same time, the gas phase of the E-line is also evacuated by keeping the cold trap (CT2) cool. Then, the dewar of liquid nitrogen at CT2 is replaced by one of pentane held at liquid-pentane freezing temperature to release trapped $\mathrm{CO}_{2}$. The pressure of the released $\mathrm{CO}_{2}$ is checked by a capacitance manometer, and the $\mathrm{CO}_{2}$ is then condensed in a Pyrex glass tube held at liquid-nitrogen temperature. When fully condensed, the glass tube is cut and sealed by standard glass-blowing techniques. Then, the dewar of pentane at CT2 is removed and cold trap CT2 is warmed to $100^{\circ} \mathrm{C}$. The pressure of released gas (mainly $\mathrm{H}_{2} \mathrm{O}$ ) is checked by a pressure transducer, and the released gas is collected into a Pyrex glass tube in the outlet part in the same way that $\mathrm{CO}_{2}$ was collected.

Gas samples, such as volcanic and geothermal gases or natural gases, are collected and held in lead-glass containers with vacuum stop cocks. ${ }^{6}$ A lead-glass container is connected with the inlet part of gas samples using a high-vacuum O-ring connector. About $1 \mathrm{~cm}^{3}$ of 


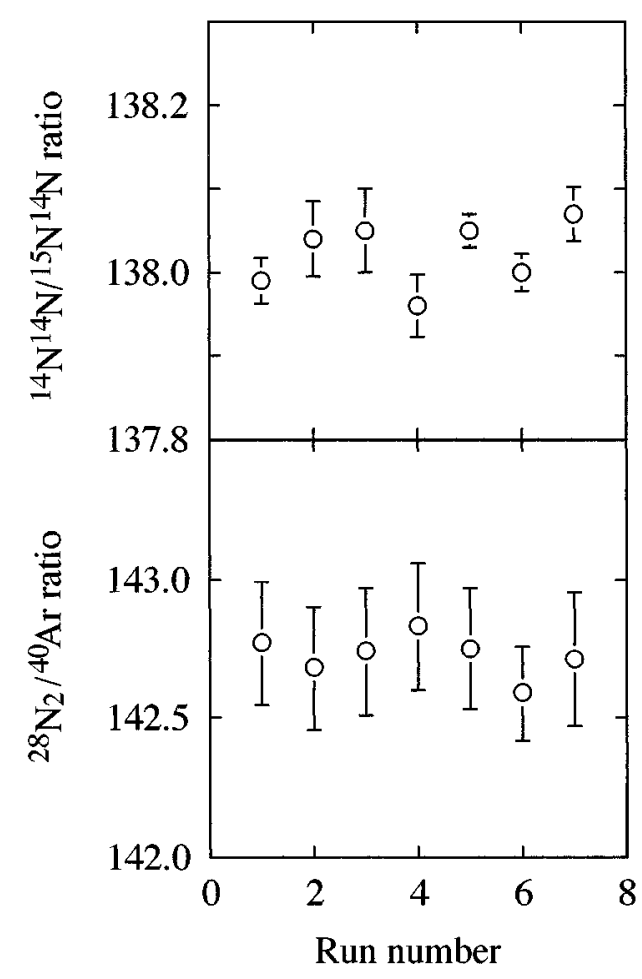

Fig. 3 Repeated measurements of 28/29 and 28/40 ratios in nitrogen standard gas. The bars indicate errors of $1 \sigma$.

a gas sample is introduced into the vacuum line by a pipette valve (PV3). At first, condensable gases, such as carbon dioxide and water vapor, are separated by a cold trap (CT5) kept at liquid-nitrogen boiling temperature. Then, the non-condensable gases are admitted into the purification line (P-line) by opening V2. After 5 min, V2 is closed; then, checking the pressure by a capacitance manometer, the quantity of gas is reduced by expansion and evacuation processes. The reason for not using a larger gas sample is to minimize the amounts of impurities, so that the gas-purification and nitrogen-separation procedures are not saturated with major volatiles. Further purification is similar to those for the rock samples described above.

\section{Mass spectrometer}

The mass spectrometer for nitrogen isotope measurement is a modified VG3600 (VG Micromass Ltd.) installed at Hiroshima University. The ion-source conditions of the mass spectrometer are optimized by using standard nitrogen gas. Nitrogen isotopes are measured at masses 28 and 29 on the single Faraday cup collector repetitively. The resolving power is about 250 at $5 \%$ of the peak height. The trap current of the ion source is $10 \mu \mathrm{A}$. The Faraday-cup sensitivity is $5.0 \mathrm{~mol} / \mathrm{A}$ for ${ }^{28} \mathrm{~N}_{2}$. The Faraday cup is equipped with a high register of $1.0 \times 10^{11} \Omega$. The detection limit is about $5 \times 10^{-15} \mathrm{~mol}$ of ${ }^{28} \mathrm{~N}_{2}$, which is equivalent to $3 \times 10^{9}$ atoms.

The major interferences at masses 28 and 29 are carbon monoxide and hydrocarbon. The interference of carbon monoxide is monitored at mass $30\left({ }^{12} \mathrm{C}^{18} \mathrm{O}\right)$, and this peak is used to check masses 28 and 29 for ${ }^{12} \mathrm{C}^{16} \mathrm{O}$ and ${ }^{13} \mathrm{C}^{16} \mathrm{O}$. After $80 \mathrm{~s}$ of waiting for equilibrium, the peaks at masses 28, 29 and 30 are scanned 15th times each. Upon completion of the 15 th scan, the computer program scans the $m / z 40$ peak so that the abundance of ${ }^{40} \mathrm{Ar}$ is measured. Then, $m / z 2,12,16,44$ are monitored. Hydrogen is monitored because excess hydrogen could produce nitrogen hydrides $\left(\mathrm{N}_{2} \mathrm{H}\right)$ which would appear at mass 29. Masses 12, 16, 44 are monitored to check the interferences of carbon monoxide and hydrocarbons at masses 28 and 29 . It takes about $20 \mathrm{~min}$ to scan all of the peaks. Mass static blanks are $6 \times 10^{-12}$ mol of ${ }^{28} \mathrm{~N}_{2}$ and $7 \times 10^{-15} \mathrm{~mol}$ of ${ }^{29} \mathrm{~N}_{2}$, and have not significant influences on measurements of the nitrogen isotopic ratio (less than $0.1 \%$ ).

The quadrupole mass spectrometer has such a performance as the mass resolving power of $2 M$ ( $M$ : mass number) and a mass range of 1 - 101 amu and a lower detection limit of $10^{-13}$ Torr. For the measurements of argon isotopes and argon/helium ratios, the intensities of ${ }^{40} \mathrm{Ar},{ }^{36} \mathrm{Ar}$ and ${ }^{4} \mathrm{He}$ are measured 50 times. At the same time, $\mathrm{m} / \mathrm{z} 3,35,37,38$ are monitored to check the interferences of hydrogen and hydrocarbons at mass 4 and 36. It takes about 7 min to scan all of the peaks. Typical analytical blanks are $6 \times 10^{-14}$ mol of ${ }^{40} \mathrm{Ar}$, $2.5 \times 10^{-16} \mathrm{~mol}$ of ${ }^{36} \mathrm{Ar}$ and $3.5 \times 10^{-15} \mathrm{~mol}$ of ${ }^{4} \mathrm{He}$.

\section{Results and Discussion}

\section{Precision of nitrogen isotope and nitrogen/argon ratios}

In order to check the analytical precision, nitrogen isotopes and nitrogen/argon ratios in standard gas (STD1) were measured repeatedly. The amount of nitrogen was about $0.15 \mathrm{nmol}$. The ${ }^{15} \mathrm{~N} /{ }^{14} \mathrm{~N}$ ratio was determined by the observed 28/29 ratio. After the standard gas was introduced into the mass spectrometer, the intensities of 28 and 29, measured 15 times, and the $28 / 29$ ratio was extrapolated at the time of gas admission into the mass spectrometer. About $1 \%$ of the nitrogen molecules decay during the measurement. After nitrogen isotope measurements, the 28/40 ratio was measured 6 times and the ${ }^{28} \mathrm{~N}_{2} /{ }^{40} \mathrm{Ar}$ ratio was determined by averaging the observed $28 / 40$ ratios. The statistical error of 15 sets of the 28/29 and 28/40 measurements was about $0.2 \%$ (which is better than that of the previous analytical system ${ }^{1}(0.5 \% \circ)$ ) and $1 \%$.

Figure 3 indicates the reproducibilities of 28/29 and $28 / 40$ ratio measurements for $0.3 \mathrm{nmol}$ aliquots of standard gas during a single day period. The overall reproducibilities are estimated to be $0.2 \%$ (which is better than that of the previous analytical system $\left.{ }^{1}(0.5 \% 0)\right)$ and $1 \%$, which are similar to the statistical errors of a single sample analysis. Under normal conditions the largest shifts in the ratio are usually confined to the first few analyses of the day when the mass spectrometer undergoes a certain amount of conditioning following a prolonged period of pumping overnight. Thus, at the start of each day, it is standard practice to admit about 
two or three consecutive aliquots of standard gas to the mass spectrometer in order to assist this stabilization process. To assess the longer term stability of the instrument, the mean 28/29 and 28/40 ratios of standard gas are plotted in Fig. 4, over the course of $20 \mathrm{~d}$. The overall reproducibilities are estimated to be $0.25 \%$ o (which is better than previous analytical system ${ }^{1}$ $(0.5 \% 0)$ ) and $1 \%$, which is similar to the errors mentioned above; therefore, the stability of the detection system during a single-day period should be such that it is not the cause of a serious error.

We tried to adjust the sample nitrogen size to be similar to that of the standard gas, as described before. However, it is difficult to make the size of the sample nitrogen exactly equal to that of the standard. Generally, a noble gas mass spectrometer in static operation shows a pressure effect on the results of the isotope ratio. That is, the isotope ratios may vary with the size of the samples. We measured 28/29 ratios of standard gas for varying amounts. Figure 5 shows the pressure effect of 28/29 ratio measurement. The ratios vary slightly with the amount of nitrogen. However, the ratio is constant within $0.1 \%$ when the sample size is adjusted to be between $0.1-0.4$ nmol. Thus, the mass spectrometer has no pressure dependence for the 28/29 ratio measurement within an uncertainty of less than $0.2 \%$ (which is better than that of a previous analytical system $\left.^{1}(1 \% o)\right)$.

We use copper oxide and platinum foils at high temperature to purify the sample nitrogen. ${ }^{7}$ To check whether the isotope ratio varies with their use, we measured the 28/29 ratios of pure nitrogen gas, whose $28 / 29$ ratio is known, in the presence of the copper oxide and platinum foils. The observed isotope ratios agree within the experimental error (Table 1). We thus found that the use of copper oxide and platinum foils does not effect the 28/29 ratio measurements.

In order to simulate an actual measurement of samples, the nitrogen isotopes and nitrogen/argon ratios of an air sample in the sample bottle were measured repeatedly. The amount of nitrogen introduced into the mass spectrometer was about $0.25 \mathrm{nmol}$. Figure 6 indicates the reproducibilities of $\delta^{15} \mathrm{~N}$ and ${ }^{28} \mathrm{~N}_{2} /{ }^{40} \mathrm{Ar}$ ratio measurements for air standard gas. The overall reproducibilities were estimated to be $0.3 \%$ (which is equivalent to previous analytical system ${ }^{1}(0.24 \% o)$ ) and $3 \%$, which is larger than the error of a standard gas analysis, therefore, we should include errors of about $0.3 \%$ and $3 \%$ for actual samples.

At the same time, the argon isotope ratios and the argon concentrations of an air sample in the sample bottle were measured by the quadrupole mass spectrometer repeatedly. The overall reproducibilities were estimated to be about $1 \%$ and $30 \%$, respectively. Also, the argon/helium ratios of another standard gas (STD2) and the $\mathrm{CO}_{2}$ concentrations of another standard sample in the sample bottle were measured repeatedly. We estimated errors of about $4 \%$ and $20 \%$ for actual samples, respectively. The $\mathrm{CO}_{2}$ blank was less than the detection limit $\left(2 \times 10^{-10} \mathrm{~mol}\right)$.

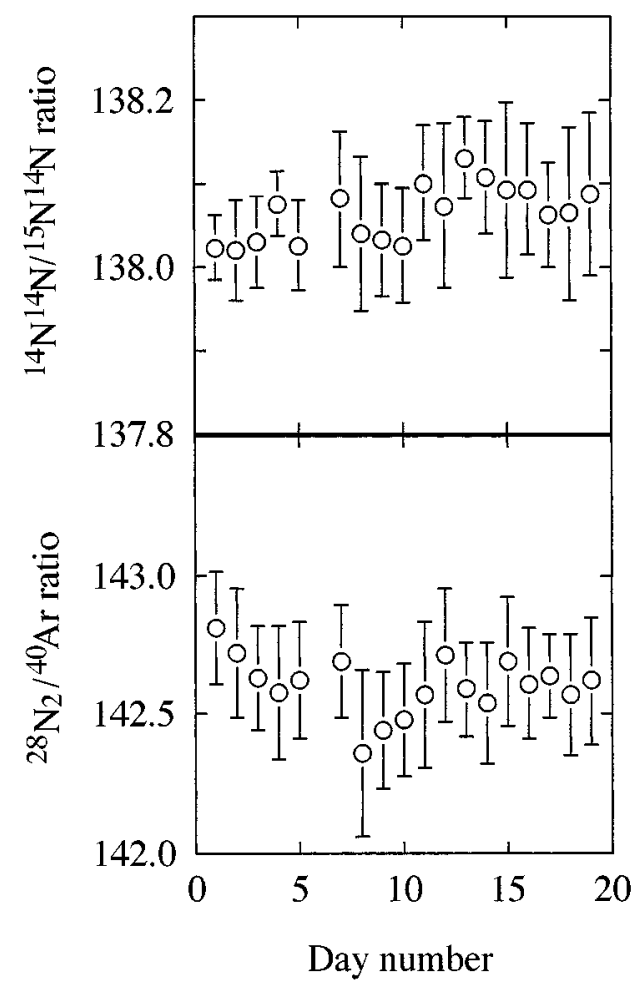

Fig. 4 Daily means of measured 28/29 and 28/40 ratios in nitrogen standard gas over a twenty-day period. The bars indicate errors of $1 \sigma$.

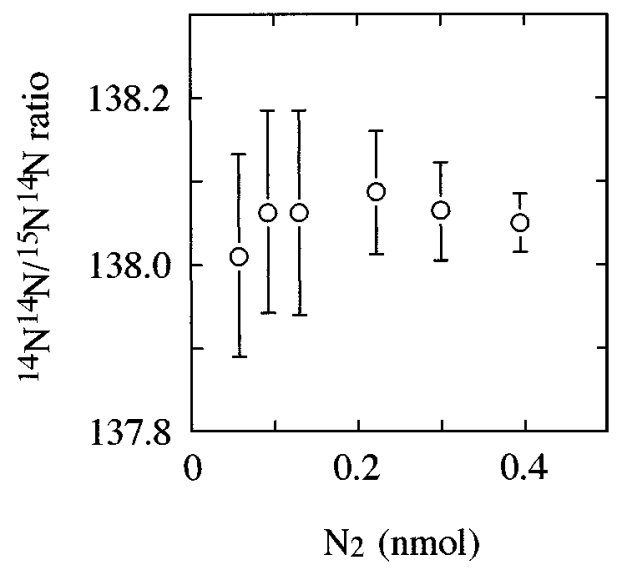

Fig. 5 Pressure dependence of 28/29 ratios in the mass spectrometer. The bars indicate errors of $1 \sigma$.

Table 1 Comparison of observed nitrogen isotopes under the presences of copper oxide and platinum foil

\begin{tabular}{ccc}
\hline $\mathrm{CuO}$ & $\mathrm{Pt}$ & $28 / 29$ ratio \\
\hline$\circ$ & $\circ$ & $138.30 \pm 0.03$ \\
$\mathrm{O}$ & $\times$ & $138.37 \pm 0.06$ \\
$\times$ & $\times$ & $138.29 \pm 0.05$ \\
\hline
\end{tabular}




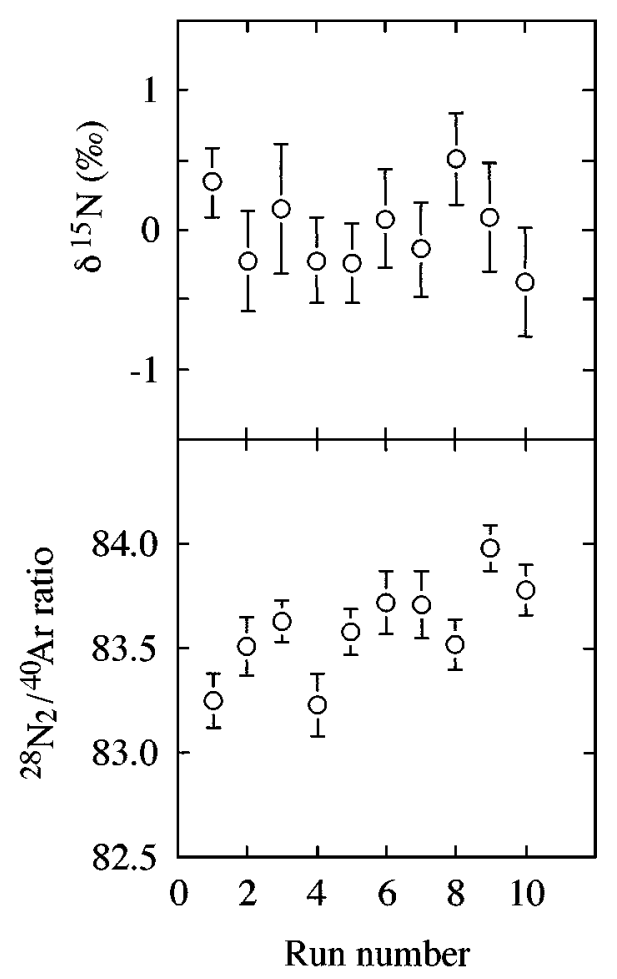

Fig. 6 Repeated measurements of 28/29 and 28/40 ratios in an air standard sample. The bars indicate errors of $1 \sigma$.

We checked the accuracy of the nitrogen isotope ratio obtained in this work by comparing our results on samples whose $\delta^{15} \mathrm{~N}$ was previously measured by a conventional dynamic mass spectrometer. Figure 7 compares the observed nitrogen isotopes and the data measured by the conventional method. According to our results given in Fig. 7, the nitrogen isotopes observed in this work agree well with reference data. The accuracy was calculated to be about $0.3 \%$ (which is better than that of the previous analytical system ${ }^{1}(0.5 \% 0)$ ).

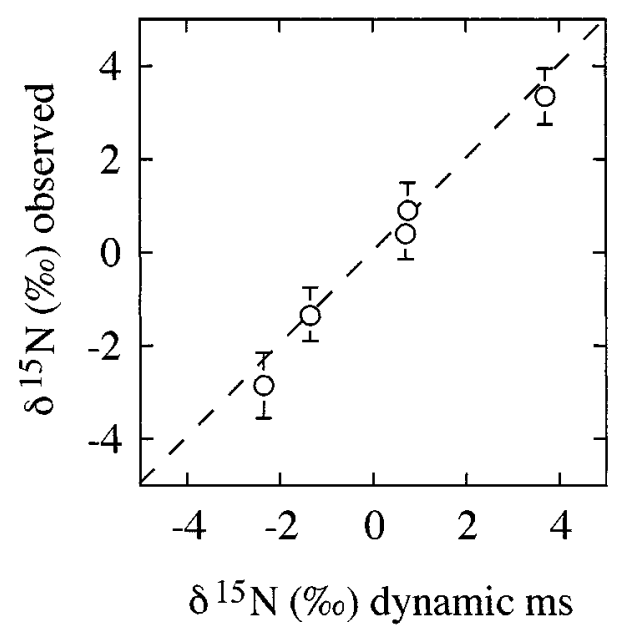

Fig. 7 Comparison of the observed nitrogen isotopes with the sample data measured by the conventional dynamic mass spectrometer. The bars indicate errors of $1 \sigma$.

\section{Isotopic measurements of terrestrial rocks}

To evaluate the ability of the instrument, we investigated six basalt glasses. Before measuring the samples, we estimated the crushing blanks by performing an experiment without a rock sample. Crushing blanks average $6 \times 10^{-12} \mathrm{~mol}$ of ${ }^{28} \mathrm{~N}_{2}$ and $4 \times 10^{-14} \mathrm{~mol}$ of ${ }^{29} \mathrm{~N}_{2}$ with an air isotopic ratio within the uncertainties, and significantly lower than the typical $\mathrm{N}_{2}$ amounts extracted from rock samples $\left(2 \times 10^{-10} \mathrm{~mol}\right)$. In most cases the contribution of the blanks is not critical for a nitrogen isotope measurement. Table 2 lists the $\delta^{15} \mathrm{~N}$ and ${ }^{28} \mathrm{~N}_{2} /{ }^{40} \mathrm{Ar},{ }^{40} \mathrm{Ar} /{ }^{36} \mathrm{Ar}$ and ${ }^{4} \mathrm{He} /{ }^{40} \mathrm{Ar}$ ratios, nitrogen concentrations and $\mathrm{C} / \mathrm{N}$ ratios of the samples. It was possible to measure the nitrogen isotope ratios within an analytical error of $0.5 \%$, which is considered to be the crushing blank. Also, four of the observed data in this study were compatible with the reported one ${ }^{2}$ within the analytical error. Thus, we found that the system is

Table $2 \delta^{15} \mathrm{~N}$, and ${ }^{28} \mathrm{~N}_{2} /{ }^{40} \mathrm{Ar},{ }^{40} \mathrm{Ar} /{ }^{36} \mathrm{Ar}$ and ${ }^{4} \mathrm{He} /{ }^{40} \mathrm{Ar}$ ratios, and nitrogen, argon and $\mathrm{CO}_{2}$ concentrations of four reference basalt glasses, which are already reported ${ }^{2}$, and of two basalt glasses

\begin{tabular}{|c|c|c|c|c|c|c|}
\hline Sample & $\begin{array}{c}\delta^{15} \mathrm{~N} \\
\% 0\end{array}$ & ${ }^{28} \mathrm{~N}_{2} /{ }^{40} \mathrm{Ar}$ & ${ }^{40} \mathrm{Ar} /{ }^{36} \mathrm{Ar}$ & ${ }^{4} \mathrm{He} /{ }^{40} \mathrm{Ar}$ & $\begin{array}{c}{ }^{28} \mathrm{~N}_{2} / \\
10^{-9} \mathrm{~mol} \mathrm{~g}^{-1}\end{array}$ & $\mathrm{C} / \mathrm{N}$ \\
\hline MAR CH31-DR11 & $-1.2 \pm 0.6^{\mathrm{a}}$ & $52^{\mathrm{a}}$ & $5190^{\mathrm{a}}$ & 11.7 & 2.5 & 2600 \\
\hline Reference & $-3.9--1.4$ & $26-63$ & $546-15029$ & & $0.04-2.2$ & 4606 \\
\hline MAR CH98-DR11 & $-3.1 \pm 0.6^{\mathrm{a}}$ & $97^{\mathrm{a}}$ & $19200^{\mathrm{a}}$ & 6.7 & 3.2 & 680 \\
\hline Reference & $-4.5--1.8$ & $84-117$ & $1237-42366$ & & $0.9-7.2$ & 418 \\
\hline EPR CLDRO1-5V & $-2.2 \pm 0.5$ & 83 & $640 \pm 8$ & 7.4 & 3.0 & 400 \\
\hline Reference & $-3.8--2.0$ & $71-74$ & $566-1850$ & & $1.0-2.4$ & 368 \\
\hline Red Sea KS-04-A & $+2.1 \pm 0.6$ & 67 & $590 \pm 19$ & 19.8 & 0.7 & 5400 \\
\hline Reference & $+2.1 \pm 0.6$ & 42 & 388 & & 0.76 & \\
\hline Woodlark \#91-655 & $+6.2 \pm 0.6$ & 95 & $290 \pm 12$ & n.d. & 3.8 & 1.3 \\
\hline Woodlark \#91-657 & $+1.3 \pm 0.7$ & 145 & $510 \pm 20$ & 0.03 & 1.7 & $<0.06$ \\
\hline
\end{tabular}

a. From Sano et al. ${ }^{8}$ n.d.: helium amount from a sample below blank. 
of practical use.

In conclusion, an analytical system for the isotopic measurement of nitrogen at the sub-nanomole level has been developed using a static mass spectrometer. The detection limit of nitrogen is about three orders of magnitude higher than that of the conventional stable isotope techniques. The analytical error of the $\delta^{15} \mathrm{~N}$ value was estimated to be about $0.3 \%$, which was better than that of previous analytical systems. ${ }^{1,5}$ We adopted the vacuum-crushing technique for nitrogen extraction, instead of the conventional stepped-heating one. Due to this, the contribution of contaminations of adsorbed air and organic materials sticking to the grain surfaces have been minimized. The simultaneous analysis of nitrogen, argon, helium, carbon dioxide and $\mathrm{H}_{2} \mathrm{O}$ abundances as well as the isotopic composition of nitrogen with high precision and argon is another advantage of this system. Previous systems ${ }^{1,5}$ could either measure only the isotopic composition of nitrogen with high precision, or could not measure nitrogen with high precision, though the noble gas ratios could be measured. Moreover, carbon dioxide extracted from the same sample used for a nitrogen isotopic measurement could be collected for $\delta^{13} \mathrm{C}$ measurement in this work.

We are grateful to H. Hiyagon, B. Marty and M. Honda for providing the basalt glass samples. We thank K. Hashizume and K. Kiyota for providing us know-hows on nitrogen purification techniques. We would like to thank H. Hirano and J. Yamamoto for their help with experiments.

This work was partly supported by Grant-in-Aid for Scientific Research Program No. 08404036 from Ministry of Education, Science and Culture of Japan.

\section{References}

1. I. P. Wright, S. R. Boyd, I. A. Franchi and C. T. Pillinger, J. Phys. E: Sci. Instrum., 21, 865 (1988).

2. B. Marty and F. Humbert, Earth Planet. Sci. Lett., 152, 101 (1997).

3. P. Cartigny, S. R. Boyd, J. W. Harris and M. Javoy, Terra Nova (in press).

4. B. Marty, Nature[London], 377, 326 (1995).

5. K. Hashizume and N. Sugiura, Mass Spectroscopy, 38, 269 (1990).

6. Y. Sano and H. Wakita, J. Geophys. Res., 90, 8728 (1985).

7. S. R. Boyd, I. P. Wright, I. A. Franchi and C. T. Pillinger, J. Phys. E: Sci. Instrum., 21, 876 (1988).

8. Y. Sano, N. Takahata, Y. Nishio and B. Marty, Geophys. Res. Lett., in press.

(Received December 15, 1997) (Accepted March 6, 1998) 